\title{
Quantitative effects of $\mathrm{Nf} 1$ inactivation on in vivo hematopoiesis
}

\author{
Youyan Zhang, ${ }^{1,2}$ Brigit R. Taylor, ${ }^{3}$ Kevin Shannon, ${ }^{3}$ and D. Wade Clapp ${ }^{1,2,4}$ \\ ${ }^{1}$ Wells Center for Pediatric Research, and \\ ${ }^{2}$ Department of Pediatrics, Indiana University School of Medicine, Indianapolis, Indiana, USA \\ ${ }^{3}$ Department of Pediatrics, University of California, San Francisco, San Francisco, California, USA \\ ${ }^{4}$ Department of Microbiology and Immunology, Indiana University School of Medicine, Indianapolis, Indiana, USA \\ Address correspondence to: D. Wade Clapp, Cancer Research Institute, 1044 West Walnut Street, Indianapolis, \\ Indiana 46202, USA. Phone: (317) 274-4719; Fax: (317) 274-8679; E-mail: dclapp@iupui.edu. \\ Youyan Zhang and Brigit R. Taylor contributed equally to this work. \\ Received for publication March 16, 2001, and accepted in revised form July 13, 2001.
}

The NF1 tumor-suppressor gene is frequently inactivated in juvenile myelomonocytic leukemia, and Nf1 mutant mice model this myeloproliferative disorder (MPD). Competitive repopulation assays were performed to quantify the proliferative advantage of $\mathrm{Nf1}^{-/-}$hematopoietic cells in vivo. $\mathrm{Nf1}$ mutant stem cells demonstrated a growth advantage that was greatest in myeloid lineage cells and least pronounced in T lymphocytes. Surprisingly, although low numbers of $N f 1$-deficient cells consistently outcompeted wild-type cells, levels of chimerism were stable over months of observation, and MPD was not observed unless threshold numbers of mutant cells were injected. These data showing that normal competitor cells can strongly modulate the growth of mutant populations in vivo have general implications for modeling cancer in the mouse. In particular, strains in which cancer-associated mutations are expressed in fields of target cells may not accurately model early events in tumorigenesis because they eliminate the requirement for a mutant clone to outcompete resident normal cells.

J. Clin. Invest. 108:709-715 (2001). DOI:10.1172/JCI200112758.

\section{Introduction}

Cancer results from a discrete series of genetic alterations in a susceptible target cell, which perturb fundamental properties such as proliferation, differentiation, survival, and responsiveness to growth-promoting or inhibitory cytokines (1). The biochemical activities of proteins implicated in tumorigenesis have largely been elucidated by a combination of in vitro experiments using purified peptides and by in vivo studies in which recombinant $\mathrm{cDNAs}$ encoding genes of interest are overexpressed in cultured cell lines. While powerful, these methodologies neither address how individual genetic lesions behave in the context of a primary cell at a specific stage of differentiation, nor do they provide quantitative data comparing the growth of mutant cells versus their normal counterparts. These questions, which have major implications both for characterizing early events in tumorigenesis and for designing rational new cancer therapies, can now be studied using cells derived from mice engineered to carry specific cancer-associated genetic lesions.

Mutations in the NF1 tumor-suppressor gene cause neurofibromatosis type 1 (NF1), a common dominantly inherited disorder. NF1 encodes neurofibromin, which binds to $\mathrm{p} 21^{\text {Ras }}$ (Ras) family members with high affinity and downregulates signaling by acting as a GTP-activating protein for Ras $(2,3)$. Individuals with NF1 are predisposed to certain cancers, including malignant peripheral nerve sheath tumor, pheochromocytoma, astrocytoma, and juvenile myelomonocytic leukemia
(JMML) (4). JMML is a relentless myeloproliferative disorder (MPD) of young children characterized by overproduction of myeloid lineage cells that infiltrate hematopoietic and nonhematopoietic tissues $(5,6)$.

Homozygous $\mathrm{N} f 1$-deficient $\left(\mathrm{Nf1^{-/ }}\right)$ mice succumb at embryonic day 12.5-13.5 (E12.5-13.5) from complex cardiac defects $(7,8)$; however, $\mathrm{Nf1}^{-/-}$fetal liver cells efficiently rescue hematopoiesis in irradiated hosts (9-11). These recipients consistently develop a JMML-like MPD with elevated myeloid cell counts, splenomegaly, and infiltration of myeloid cells into the liver and spleen (9-11). Genetic and biochemical studies of human and murine hematopoietic cells have shown that $N f 1$ functions as a tumor suppressor through the ability of neurofibromin to negatively regulate Ras output $(9,12-15)$. Hypersensitivity to GM-CSF plays a central role in the aberrant growth of human JMML and murine $N f 1$-deficient cells $(6,9,14)$. In addition, $N f 1$ deficient cells form excessive numbers of progenitor colonies in response to stem cell factor (SCF), and recent studies have demonstrated abnormal signaling from its receptor, the c-kit tyrosine kinase, in heterozygous Nf1 mutant mast cells $(10,16,17)$.

While much has been learned about how inactivation of $N f 1$ deregulates hematopoietic growth, important questions remain. First, the quantitative effects of $N f 1$ loss on the growth of hematopoietic cells are unknown. Second, although adoptive transfer studies in mice suggest that inactivation of $N f 1$ is both necessary and sufficient to induce MPD in irradiated recipients, these stud- 
ies do not account for the potential effects of coexisting normal cells on the growth of $\mathrm{Nf1}^{-/-}$stem cells within the marrow microenvironment. Finally, data from patients with JMML suggest that the disease originates in a cell with the capacity to differentiate into myeloid or lymphoid progeny in at least some instances $(18,19)$. This is certainly true in murine recipients repopulated with $\mathrm{Nf1}^{--}$fetal liver cells, in which the entire hematopoietic system is derived from mutant cells. Despite this, the hematologic disorders that arise in children with NF1 and in murine recipients of $\mathrm{Nf}^{-/-}$fetal liver cells are almost invariably myeloid. These observations suggest a differential requirement for neurofibromin in myeloid versus lymphoid growth control, and it remains unclear whether neurofibromin modulates $\mathrm{p} 21^{\text {ras }}$ GTPase activity in other hematopoietic lineages $(20,21)$.

The hematopoietic system is hierarchical in nature, well-established in vitro assays exist for measuring the growth of precursors derived from discrete compartments, and the technique of competitive repopulation permits direct in vivo comparisons between mutant and wild-type cells in a variety of lineages. Hematopoiesis therefore provides a model experimental system for quantifying the effects of specific cancer-associated mutations such as $N f 1$ inactivation in differentiating primary cells. We performed competitive repopulation studies to compare the growth of $\mathrm{Nf1}^{-/-}$hematopoietic cells to that of normal cells in vivo. We hypothesized that Nf1 mutant cells would consistently outcompete wild-type cells and that this growth advantage would increase over time such that all recipients engrafted with mutant cells would ultimately develop MPD. We further hypothesized that inactivation of $N f 1$ would confer a strong proliferative advantage in myeloid cells, and we set out to determine whether this was also seen in the stem cell compartment and in lymphoid cells.

Here we show that the genetic background of Nf1deficient donor cells influences the phenotype of the MPD that develops after adoptive transfer. Furthermore, inactivation of $N f 1$ results in differential expansion of immature progenitors in the spleens and bone marrows of irradiated recipients. We also find that Nf1deficient cells demonstrate a relatively modest growth advantage over normal cells that is more pronounced in, but is not restricted to, the myeloid compartment. Surprisingly, Nf1-deficient cells did not progressively outcompete their wild-type counterparts over months of observation. Instead, we found stable chimerism in irradiated recipients. Furthermore, MPD only developed in recipients that received threshold numbers of Nf1 mutant cells. These data suggest that genetically engineered murine cancer models that create fields of mutant cells may not recapitulate all of the mutational events that are required for clonal outgrowth from a single mutant precursor.

\section{Methods}

Progenitor assays. Colonies derived from myeloid progenitors were enumerated exactly as described (10).
Recombinant murine GM-CSF, IL-3, and SCF were purchased from PeproTech Inc. (Rocky Hill, New Jersey, USA), and recombinant IL-1 and M-CSF were obtained from R \& D Systems Inc. (Minneapolis, Minnesota, USA). Cells and recombinant growth factors were added to agar for growth of low proliferating potential colony- forming cell (LPP-CFC) and high proliferating potential-colony forming cell (HPP-CFC) and the solution thoroughly admixed before plating. LPP-CFC and HPP-CFC were enumerated in $8 \% \mathrm{CO}_{2}$ and $5 \% \mathrm{O}_{2}$. LPP-CFC and HPP-CFC were scored by indirect microscopy on days 7 and 14 , respectively.

Purification of Sca $1^{+}$lin-/dim cells. Low-density mononuclear cells from littermates of each genotype were pooled prior to cell sorting and resuspended in cold PBS $0.1 \%$ BSA at a concentration of 1 to $2 \times 10^{8}$ cells $/ \mathrm{mL}$. The cells were stained for 20 minutes at $4^{\circ} \mathrm{C}$ with the following Ab's: Sca1-phycoerythrin (PE), CD3-FITC, B220-FITC, Mac1-FITC, and Gr1-FITC (PharMingen, San Diego, California, USA). Samples were washed twice and resuspended at a concentration of 5 to $10 \times 10^{6}$ cells $/ \mathrm{ml}$ cold PBS $0.1 \%$ BSA. The cells were sorted for Sca $1^{+}$lin-/dim cells using a FACSTAR sorter (Becton Dickinson, San Diego, California, USA) employing identical sorting gates.

Competitive repopulation experiments. This procedure has been reported elsewhere (22). Female C57Bl/6J mice that were lethally irradiated (1,100 cGy split dose) received limiting dilutions of $0-10,000 \mathrm{Sca}^{+} \mathrm{lin}^{-/ \mathrm{dim}}$ cells isolated from either $\mathrm{Nf1}^{-/-}$or $\mathrm{Nf1}^{+/+}$fetal livers cells mixed with $5 \times 10^{5}$ B6.BoyJ low-density competitor cells by tail vein injection. Six irradiated recipients were injected at each test cell concentration. Control groups received either BoyJ competitors alone or no cells. Four independent experiments were performed.

Adoptive transfer of unsorted fetal liver cells. Approximately one million d12.5-13.5 $\mathrm{Nf1}^{+/+}$or $\mathrm{Nf1}^{-/-}$fetal liver cells from intercrossed $\mathrm{Nf1}^{+/-}$mice on a C57Bl/6J (at least 10 generations) or $129 / \mathrm{Sv}$ inbred strains (generated from $129 /$ Sv embryonic stem cells) were transplanted into the respective strains of syngeneic recipients following irradiation with 1,100 cGy. C57Bl6/129Sv F1 $\mathrm{Nf1}^{-/-}$and $\mathrm{Nf1^{+/+ }}$ d13.5 fetal liver cells were transplanted into C57Bl6/129Sv F1 recipients or into 129/Sv recipients to examine strain effects of the myeloproliferative disease. Recipients were evaluated sequentially to confirm that signs of graft-versus-host disease, such as runting, hair loss, and diarrhea, were absent.

Chimerism analysis by fluorescence cytometry. Tail vein blood samples $(100 \mu \mathrm{L})$ were analyzed for CD45.1/2 chimerism and with multilineage markers as described elsewhere (22). A total of 5,000 events were collected from each sample. All data were analyzed using CELLQuest software (Becton Dickinson, San Diego, California, USA). Instrument settings and gates used to analyze data were identical from month to month and between genotypes. An unpaired Student's $t$ test was used to determine whether significant differences existed in chimerism between genotypes. 
Repopulating unit calculation. A formula described by Harrison et al. (23) was used to compare the repopulating abilities of donor-versus-competitor cells. This calculation allows quantitative comparison of repopulating ability between different donor cell populations (i.e., $\mathrm{Nf1}^{-/-}$and $\mathrm{Nf1}^{+/+}$). We calculated the repopulating units (RUs) for mice transplanted with the highest dose of Sca $1^{+} \mathrm{lin}^{-/ \mathrm{dim}}$ cells. Since a constant number of competitors $\left(5 \times 10^{5}\right)$ were used for all experiments, the following equation was used to compute RUs: $\mathrm{RU}=5 \times$ measured donor chimerism/(100 measured donor chimerism). Differences in RUs between groups were compared using an unpaired Student's $t$ test.

\section{Results}

Effects of strain background on MPD. We crossed the Nf1 mutation onto a C57BL/6 background for 10 generations in order to perform competitive repopulation experiments in congenic mice (see below). Unexpectedly, the background of the donor fetal liver cells modulated the phenotype of the MPD in irradiated hosts. Syngeneic recipients transplanted with $129 / \mathrm{Sv} \mathrm{Nf1}^{-/-}$ fetal liver cells had modestly elevated total leukocyte and myeloid cell counts 2 months after adoptive transfer and showed marked increases by 4 months (Figure 1a). Recipients of C57BL/6 $\mathrm{Nf1}^{-/-}$cells showed lower blood counts at these times (Figure 1a). The genetic background of donor fetal liver cells had more striking effects on survival and splenomegaly. Mice transplanted with $129 / \mathrm{Sv}$ cells showed substantial mortality by 6 months (Figure 1b); this was associated with splenomegaly (Figure 1c) and with myeloid cell infiltration within the liver and spleen (data not shown). Although recipients of $\mathrm{Nf1}^{-1-} \mathrm{C} 57 \mathrm{BL} / 6$ fetal liver cells uniformly developed MPD, the course was more indolent with enhanced survival and smaller spleen size (Figure 1, b and c). Approximately $90 \%$ of these recipients survived to at least 1 year of age (data not shown). Heterozygous Nf1 mutant 129/Sv and C57BL/6 mice were mated to ascertain which hematopoietic phenotype is dominant. Recipients injected with $\mathrm{F}_{1}$ fetal liver cells developed markedly elevated total peripheral white blood cell and myeloid cell counts (Figure 1d), prominent splenomegaly, and early mortality that was at least as severe as the MPD seen in the inbred 129/Sv background. Together, the results presented in Figure 1 indicate that crossing the Nf1 mutation onto the C57BL/ 6 background attenuates the MPD that arises in recipients of Nf1-deficient fetal liver cells.

Organ-specific expansion of immature Nf1-deficient progenitors in vivo. $\mathrm{Nf1}^{-1-}$ fetal liver cells form increased numbers of colonies derived from both primitive progenitors with significant replating potential, referred to as HPP-CFC, and from more mature lineage restricted myeloid progenitors called LPP-CFC (10). Since splenomegaly is a significant component of both JMML and of the MPD seen in mice transplanted with $\mathrm{Nf1}^{-/-}$cells, we compared LPP-CFC and HPP-CFC num- bers in the bone marrows and spleens of recipients of C57BL/ 6 fetal liver cells. Absolute numbers of progenitors per organ were calculated by multiplying the frequency of each progenitor detected in two-layer agar cultures by the number of low-density cells per organ. The bone marrows of mice that received $\mathrm{Nf}^{-/-}$cells showed a marked increase in the numbers of LPP-CFC progenitor cells compared with recipients of wild-type cells, while absolute numbers of more primitive hematopoietic HPP-CFC progenitors were equivalent (Figure 2). Thus, expansion of $\mathrm{Nf}^{-/-}$cells occurs at the level of relatively mature and differentiated progenitors in recipient marrow. In contrast, there was marked expansion of both HPP-CFC and LPP-CFC compartments in the spleens of mice transplanted with $\mathrm{Nf1}^{-/-}$ cells. The bone marrows and spleens of recipients engrafted with $\mathrm{Nf1}^{-/-}$fetal liver cells also showed a marked expansion in the numbers of burst-forming
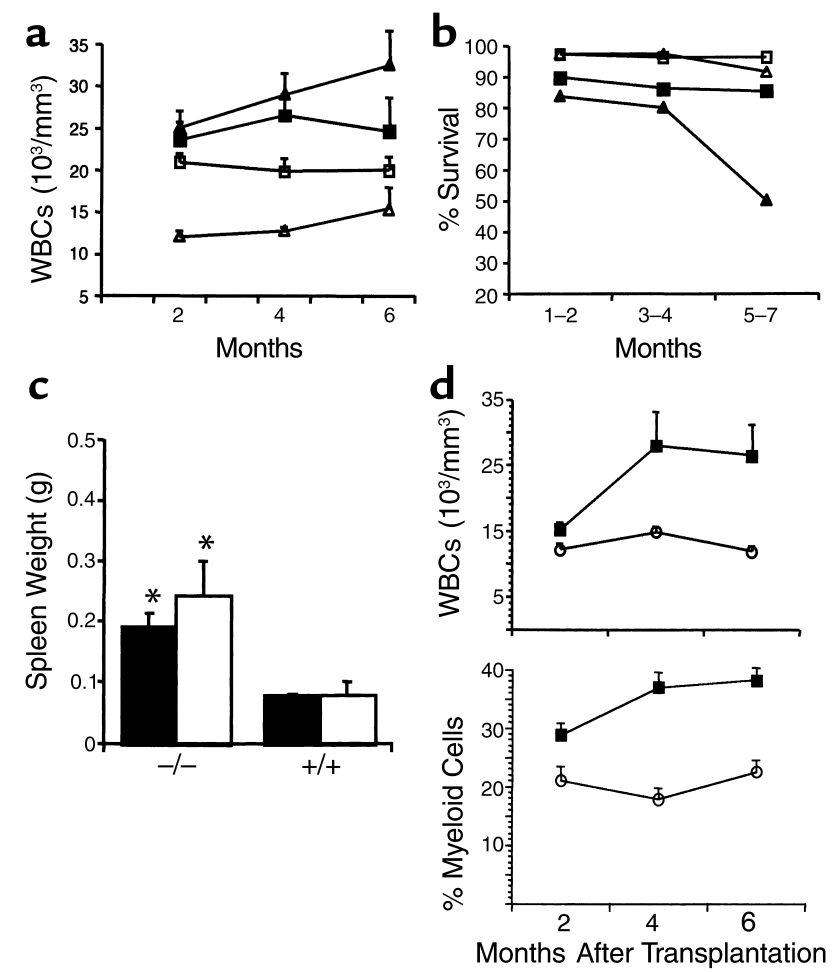

Figure 1

Leukocyte counts, spleen sizes, and survival in recipients of $\mathrm{Nf1}^{-/-}$ fetal liver cells from C57BL/6, 129/Sv, or $F_{1}$ embryos. ( $\mathbf{a}$ and $\mathbf{b}$ ) Recipients injected with wild-type C57BL/ 6 fetal liver cells are denoted by open squares, and recipients injected with $N f 1^{-/-} \mathrm{C} 57 \mathrm{BL} / 6$ cells are denoted by filled squares. Recipients that received wild-type or $N f 1^{-1-} 129 /$ Sv fetal liver cells are denoted by open and filled triangles, respectively. Leukocyte counts and percentage of survival 2-6 months after adoptive transfer. (c) Spleen weights at sacrifice. Filled bars represent C57BL/6. Open bars represent $129 / \mathrm{Sv}$. ${ }^{*} P<0.05$ comparing $^{-/-}$to ${ }^{+/+}$spleen weight within the same donor strain. (d) Total leukocyte (WBC) and myeloid cell counts of recipient mice reconstituted with either $\mathrm{Nf1^{-- }}$ (squares) or wild-type (circles) fetal

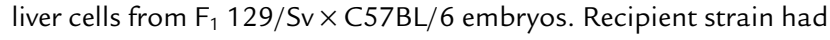
no effect on WBC counts, survival, or spleen size (data not shown). WBC, white blood cell. 

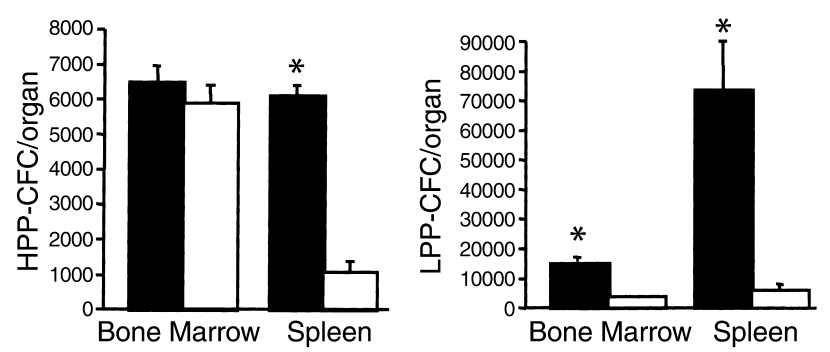

Figure 2

LPP and HPP progenitors in bone marrow and spleen. Growth of LPP and HPP colonies from the bone marrows and spleens of recipients transplanted with $\mathrm{Nf1}^{-1-}$ (filled bars) or with wild-type (open bars) fetal liver cells. Total numbers of LPP-CFCs and HPP-CFCs per organ are shown. ${ }^{*} P<0.01$ comparing $N f 1^{-/-}$vs. $N f 1^{+/+}$.

unit erythroid (BFU-E) and colony-forming unit granulocyte, macrophage, erythroid, and megakaryocyte (CFU-GEMM) progenitors (data not shown).

Quantitative growth advantage of $\mathrm{Nf1}^{-1-}$ hematopoietic cells. The competitive repopulation assay provides a way of quantifying the effects of a specific mutation on the growth of cells within multiple hematopoietic compartments (23). This assay is sensitive to small differences in test cell populations because the entire differentiation pathway is analyzed. FACS was performed on fetal liver cells from $\mathrm{Nf}^{-/-}$and $\mathrm{Nf}^{+/+}$littermates to isolate Sca1 ${ }^{+} \mathrm{lin}^{-/ \mathrm{dim}}$ cells, a fraction that is highly enriched for stem and progenitor cells. A range of test cell numbers of each genotype was mixed with $5 \times 10^{5}$ congenic competitor cells from a strain (C57BL/6 BoyJ) that expresses an isoantigen of the pan-hematopoietic cell surface marker CD45. These cells were transferred into irradiated hosts, and sequential peripheral blood samples collected from these recipients over 6 months were analyzed by FACS. As expected, the total CD45.1 (BoyJ) and CD45.2 (test cell) chimerism of individual animals approximated $100 \%(100 \% \pm 1 \%$, data not shown). Recipients transplanted with $\mathrm{Nf1}^{-/-}$fetal liver cells had a two- to threefold higher test cell chimerism over a 100 -fold range of input test cells relative to wild-type cells (Figure 3a). To determine which lineages are affected by inactivation of $N f 1$, we performed two-color staining using anti-CD45.2 and a series of Ab's that are specific to myeloid or lymphoid lineages. Although recipients engrafted with $N f 1$-deficient fetal liver cells consistently maintain normal lymphocyte counts and do not develop lymphoid malignancies (9-11), $\mathrm{Nf1}^{-/-}$ cells achieved a growth advantage in both myeloid and lymphoid lineages (Figure 3, b-e). However, there were quantitative differences in the proliferative advantage of $\mathrm{Nf1}^{-/-}$cells between myeloid and lymphoid compartments. For example, while adoptive transfer of 250 Sca $1^{+} \mathrm{lin}^{-/ \mathrm{dim}}$ cells resulted in approximately 50\% chimerism in granulocytes and macrophages, equivalent chimerism in $\mathrm{T}$ lymphocytes required transplantation of 1,000 Sca1 $1^{+}$lin ${ }^{-/ d i m}$ cells (Figure 3, b-d). $\mathrm{Nf1}^{-/-}$ $\mathrm{B}$ lymphocytes had a proliferation advantage over
$\mathrm{Nf1}^{+/+}$cells that was similar overall to the advantage observed in the myeloid lineages (Figure 3e). Serial analyses using an established measure of repopulating ability (24) demonstrated that the proliferative advantage of Nf1-deficient cells was stable over 6 months of observation (Figure 3f). Finally and importantly, there was a clear threshold effect with respect to the number of $\mathrm{Nf}^{-/-} \mathrm{Sca}^{+}{ }^{+} \mathrm{lin}^{-/ \mathrm{dim}}$ fetal liver cells transferred and the development of MPD. Recipients that received less than 1,000 test cells in four independent experiments maintained normal leukocyte and myeloid cell counts during 6 months of observation (Figure 3g) and did not develop enlarged spleens nor have evidence of disease. By contrast, recipients injected with 1,000-5,000 Nf1-deficient test cells consistently developed overt MPD with elevated leukocyte counts and an increased percentage of myeloid cells (Figure 3g). These mice also developed marked splenomegaly (mean spleen weight $310 \pm 68 \mathrm{mg}$ SEM) and showed reduced survival comparable to recipients transplanted with $\mathrm{Nf1}^{-/-}$cells only.

Enhanced growth of $\mathrm{Nf1}^{-/-}$cells after secondary transfer. The observation that $\mathrm{Nf1}^{-/-}$cells outcompete their wildtype counterparts in all hematopoietic lineages provided indirect evidence of a growth advantage within the stem cell compartment. To test this idea further, bone marrow cells from primary recipients of fetal liver cell transplants showing a 50\% level of chimerism in blood leukocytes were transferred into secondary hosts, and chimerism was assessed 4 months later. A stable level of CD45.1/CD45.2 chimerism was observed when cells from primary hosts that had received test and competitor cells collected from wild-type fetal livers were transferred. In contrast, secondary recipients transplanted with bone marrow cells from primary hosts that had initially received Nf1-deficient test cells consistently showed a substantial increase in the percentage of CD45.2-positive leukocytes derived from

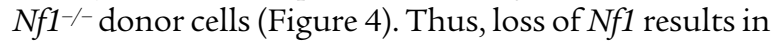
an increase in stem cell repopulating ability.

\section{Discussion}

Adoptive transfer of $\mathrm{Nf1}$-deficient fetal liver cells into irradiated recipients provides a model of JMML (9) that has been used for biologic and preclinical studies $(10,11$, 25). One finding from this study is that the most immature population of progenitors assayed, the HPP-CFC, was markedly expanded in the spleens of recipient mice but is normal in the marrow. This suggests that the marrow microenvironment does not support expansion of the most immature $\mathrm{Nf1}^{-/-}$progenitors, while the spleen is permissive for overgrowth in the HPP-CFC compartment. These data may be relevant to the pathogenesis of human JMML, which is frequently characterized by massive splenomegaly $(5,6)$.

We found that crossing the Nf1 mutation onto a C57BL/6 background modulated the phenotype associated with gene inactivation. Data from the $\mathrm{F}_{1}$ cross between C57BL/ 6 and 129/Sv revealed that the 129/Sv background dominantly enhances the severity of the 

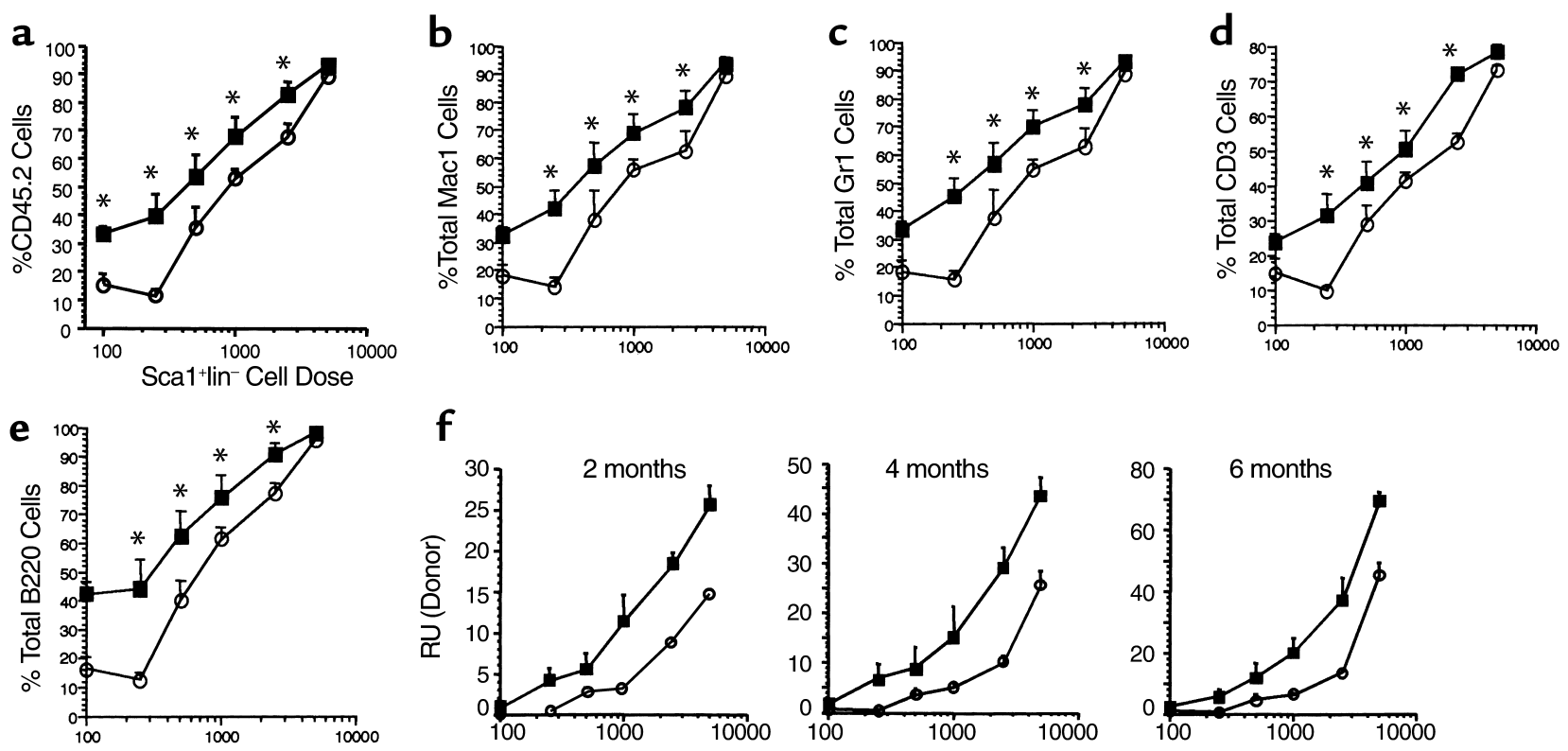

g

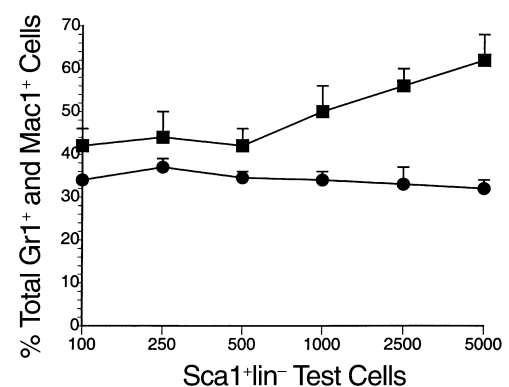

Figure 3

Comparative growth of $\mathrm{Nf1}^{-/-}$and wild-type Sca $1^{+} \operatorname{lin}{ }^{-/ \mathrm{dim}}$ cells in competitive repopulation assays. $\mathrm{Nf1^{-/ }}$ cells are shown as squares and wild-type cells as circles. (a-e) Test cell chimerism in blood leukocytes (CD45.2-positive), monocytes and macrophages (Mac1-positive), granulocytes (Gr1-positive), T lymphocytes (CD3-positive), and B lymphocytes (B220-positive). ${ }^{*} P<0.05$ comparing $N f 1^{-/-}$vs. $N f 1^{+/+}$. (f) Comparative repopulating abilities of $N f 1^{-/-}$and wild-type cells over time. (g) Evaluation of the proportion of myeloid and lymphoid cells in recipients reconstituted with 100-5,000 Sca $1^{+} \operatorname{lin}^{-/ \text {dim }}$ test cells.

MPD caused by inactivation of $N f 1$. An $\mathrm{F}_{2}$ cross will be required to ascertain if this is encoded by a single locus or is due to a number of interacting modifiers. Interestingly, male sex and maternal transmission of a mutant NF1 allele influence the risk of developing myeloid leukemia in children with NF1 $(26,27)$. Our data, which underscore the importance of accounting for genetic background in characterizing murine models of human cancer, are consistent with a recent study in which strain background modified the incidence of glioblastoma in a line of mice that carried mutations of both $N f 1$ and $p 53$ in cis (28).

While the competitive repopulation assay is commonly viewed as a sensitive way of measuring the long- term proliferative potential of hematopoietic stem cells, our data indicate that the growth advantage of mutant cells varies in a lineage-specific manner. Inactivation of $N f 1$ confers a growth advantage that is most pronounced in the myeloid compartment and less apparent in T lymphocytes. We also performed secondary adoptive transfer experiments to prove that that $\mathrm{N}^{1^{-/}}$stem cells outcompete wild-type cells. Our data are consistent with previous studies showing that both children with NF1 and heterozygous Nf1 mutant mice are strongly predisposed to myeloid, but not lymphoid, malignancies $(9,26,29,30)$. Data from our laboratories and others implicate differential responses of the progeny of mutant stem cells to extracellular
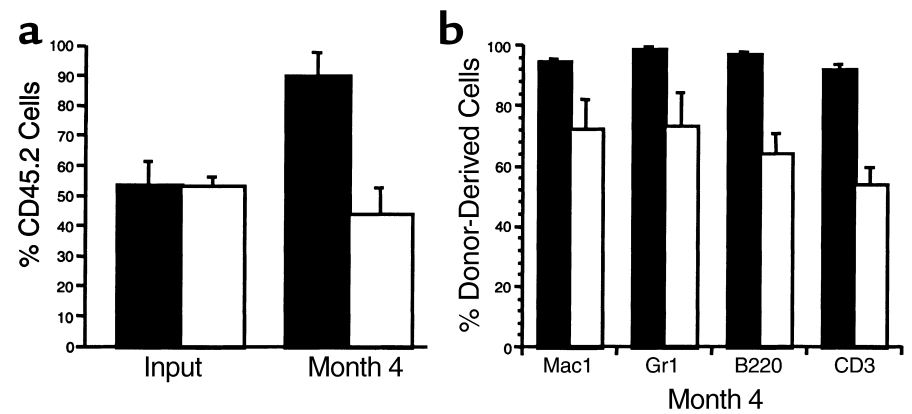

Figure 4

Chimerism of secondary recipients. Total test cell chimerism (a) and lineage-specific chimerism (b) is shown for recipients that received bone marrow cells from primary recipients showing 50:50 CD45.1/45.2 chimerism. Mice were transplanted from primary recipients that received $\mathrm{Nf1}^{-/-}$(filled bars) or wild-type (open bars) fetal liver test cells, respectively. 


\section{Figure 5}

Proposed model for spontaneous development of MPD/JMML. Loss of the normal Nf1 allele in an early hematopoietic cell (dark cell) initiates leukemia development. One (or more) cooperating genetic event is required to permit this clone to outcompete normal stem cells in the marrow microenvironment. This mutant population induces clinical disease, which is driven by aberrant growth in response to GM-CSF and to other myeloid growth factors that signal through Ras.

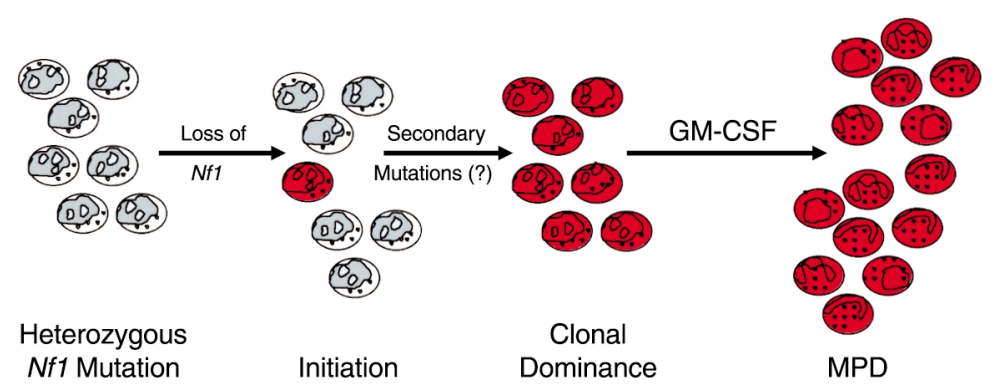

stimuli such as growth factors, which confer a growth $(9,10)$ and survival $(16,31)$ advantage to $N f 1$ mutant hematopoietic cells. These cellular phenotypes might be due to an alteration in the threshold responsiveness of cells to basal levels of cytokine growth factors that signal through p2 $1^{\text {ras }}-$ GTP (17). Expression of the $B C R-A B L$ fusion oncogene and inactivation of the tumor suppressor Pten are thought to perturb growth, in part, by sensitizing cells to growth factors $(32,33)$. Furthermore, hyper-responsiveness to GM-CSF and, perhaps, to SCF and other myeloid growth factors is implicated as playing a central role in human JMML and in the MPD that develops in recipients of $\mathrm{Nf1}^{-/-}$ transplants $(6,11,34)$. In vitro data indicate that $N f 1$ deficient hematopoietic cells have increased proliferation and survival in response to multiple growth factors. While mice transplanted with only $\mathrm{Nf1}^{-/-}$fetal liver cells invariably develop MPD, and $\mathrm{Nf1}^{-/-}$cells outcompete wild-type cells in competitive repopulation experiments, it is unclear whether in vivo expansion of myeloid cells is secondary to increased proliferation and/or survival. Preliminary data, based on FACS analysis of phenotypically identified progenitor/stem cell populations, indicate that there are more $\mathrm{Nf1}^{-/-}$ cells in S-phase as compared with wild-type cells and that $\mathrm{Nf1}^{-\gamma^{-}}$cells are significantly more resistant to apoptosis (unpublished results, D. Ingram and W. Clapp). Studies are currently underway to determine the biochemical mechanisms for these phenotypes and to determine the relative role of each of these cellular functions in contributing to the myeloproliferative disease observed in mice transplanted with $\mathrm{Nf}^{-/-}$cells. We were surprised to discover that low numbers of Nf1-deficient $\mathrm{Sca}^{+}{ }^{+}$lin$^{-} /$dim engrafted readily in the stem cell compartment and achieved a growth advantage over wild-type cells in all differentiated lineages tested, but this did not invariably evolve to MPD. Instead, threshold numbers of $\mathrm{Nf}^{-/-}$cells were required to induce the JMML-like myeloid disorder. The stable levels of chimerism observed over 6 months of observation are unlikely to be explained by a slow rate of stem cell turnover because murine stem cells replicate approximately once every 2.5 weeks in vivo (35). Instead, our data imply that a steady state is achieved between Nf1deficient and wild-type cells in recipient bone marrows. This may be similar to human patients who have been successfully treated for myeloid leukemia associated with a translocation $t(8 ; 21)$, many of whom continue to express a leukemia-associated fusion mRNA for many years (36). The capacity of normal cells within the marrow microenvironment to modulate the fate of mutant precursors was shown recently in a study in which an intrinsic defect erythroid differentiation in cells overexpressing the GATA-1 transcription factor was rescued in a cell nonautonomous manner (37). We have shown recently that heterozygous inactivation of $N f 1$ rescues many of the phenotypic and biochemical defects found in White spotted locus $(W)^{41}$ mutant mast cells (16). Similarly, it will be interesting to determine if haploin sufficiency for $N f 1$ confers a growth advantage in hematopoietic stem cells.

The absence of MPD in mice that received fewer than $1,000 \mathrm{Nf1}^{-/-} \mathrm{Sca} 1^{+} \mathrm{lin}^{-/ \text {dim }}$ fetal liver cells implies that cooperating mutations might be required for induction of MPD in a marrow microenvironment that is mostly comprised of wild-type cells. This idea is consistent with the relatively long latency before the onset of myeloid malignancy in untreated and cyclophosphamideexposed heterozygous $N f 1$ mutant mice $(8,25)$. Furthermore, somatic inactivation of $N f 1$ was not invariably associated with evidence of MPD in the cyclophosphamide study (25). These data are also compatible with human data showing that cooperating genetic events such as loss of chromosome 7 are often found in addition to inactivation of NF1 in the bone marrows of children who develop myeloid malignancies $(26,38,39)$.

Murine cancer models frequently rely on either overexpressing an oncogene product or deleting a tumor suppressor in a field of target cells. How accurately this reflects human tumorigenesis, which is generally thought to involve the sequential acquisition of mutations in progeny derived from a single mutant precursor cell, is unknown. In particular, the potential inhibitory effect of normal cells within tissue microenvironments on the proliferation of mutant cells has received limited attention. We have shown that inactivating $N f 1$ in hematopoietic stem cells only results in MPD if threshold numbers of mutant cells are injected. These data infer that tumor initiation and clonal outgrowth are distinct events (Figure 5). In leukemias that arise spontaneously from a single aberrant precursor, it is likely that cooperating genetic lesions are required for clonal dominance (Figure 5). Our data suggest that transgenic models in which tumors derived from fields 
of mutant cells may obviate the requirement for one or more early cooperating genetic lesions that facilitate clonal outgrowth in vivo. If this is true, caution should be exercised in extrapolating data regarding tumor biology and preclinical responses to experimental therapeutics from these model systems to human cancers.

\section{Acknowledgments}

We are indebted to Tyler Jacks for providing Nf1 mice and for valuable discussions. We also thank Mervin Yoder and Laura Haneline for their comments during the preparation of the manuscript. This work was supported by American Cancer Society grant LBC-98582, by FIRST award R29 CA74177 to D.W. Clapp, and by R01 CA72624 to K. Shannon.

1. Hanahan, D., and Weinberg, R.A. 2000. The hallmarks of cancer. Cell. 100:57-70.

2. Boguski, M.S., and McCormick, F. 1993. Proteins regulating Ras and its relatives. Nature. 366:643-654.

3. Bernards, A. 1995. Neurofibromatosis type 1 and Ras-mediated signaling: filling in the GAPs. Biochim. Biophys. Acta. 1242:43-59.

4. Side, L., and Shannon, K. 1998. The NF1 gene as a tumor suppressor. In Neurofibromatosis type 1. M. Upashyaya and D. Cooper, editors. Bios Scientific Publishers Ltd. Oxford, United Kingdom. 133-152.

5. Arico, M., Biondi, A., and Pui, C.H. 1997. Juvenile myelomonocytic leukemia. Blood. 90:479-488.

6. Emanuel, P., Shannon, K., and Castleberry, R. 1996. Juvenile myelomonocytic leukemia: molecular understanding and prospects for therapy. Mol. Med. Today. 2:468-475.

7. Brannan, C.I., et al. 1994. Targeted disruption of the neurofibromatosis type-1 gene leads to developmental abnormalities in heart and various neural crest-derived tissues. Genes Dev. 8:1019-1029.

8. Jacks, T., et al. 1994. Tumorigenic and developmental consequences of a targeted Nf1 mutation in the mouse. Nat. Genet. 7:353-361.

9. Largaespada, D.A., Brannan, C.I., Jenkins, N.A., and Copeland, N.G. 1996. Nf1 deficiency causes Ras-mediated granulocyte/macrophage colony stimulating factor hypersensitivity and chronic myeloid leukaemia. Nat. Genet. 12:137-143.

10. Zhang, Y.Y., et al. 1998. Nf1 regulates hematopoietic progenitor cell growth and ras signaling in response to multiple cytokines. J. Exp. Med. 187:1893-902.

11. Birnbaum, R.A., et al. 2000. $N f 1$ and Gmcsf interact in myeloid leukemogenesis. Mol. Cell. 5:189-195.

12. Shannon, K., et al. 1994. Loss of the normal NF1 allele from the bone marrow of children with type 1 neurofibromatosis and malignant myeloid disorders. N. Engl. J. Med. 330:597-601.

13. Kalra, R., Paderanga, D.C., Olson, K., and Shannon, K.M. 1994. Genetic analysis is consistent with the hypothesis that NF1 limits myeloid cell growth through p21ras. Blood. 84:3435-3439.

14. Bollag, G., et al. 1996. Loss of NF1 results in activation of the Ras signaling pathway and leads to aberrant growth in hematopoietic cells. Nat. Genet. 12:144-148.

15. Side, L., et al. 1997. Homozygous inactivation of the NF1 gene in bone marrow cells from children with neurofibromatosis type 1 and malignant myeloid disorders. N. Engl. J. Med. 336:1713-1720.

16. Ingram, D.A., et al. 2000. Genetic and biochemical evidence that hap- loinsufficiency of the $N f 1$ tumor suppressor gene modulates melanocyte and mast cell fates in vivo. J. Exp. Med. 191:181-188.

17. Ingram, D.A., et al. 2001. Hyperactivation of 2 21ras and the hematopoietic specific Rho GTPase, Rac2, cooperate to alter the proliferation of neurofibromin deficient mast cells in vivo and in vitro. J. Exp. Med. 194:57-70.

18. Lau, R.C., et al. 1994. Lymphoid blast crisis of B-lineage phenotype with monosomy 7 in a patient with juvenile chronic myelogenous leukemia (JCML). Leukemia. 8:903-908.

19. Cooper, L.J., et al. 2000. Evidence that juvenile myelomonocytic leukemia can arise from a pluripotential stem cell. Blood. 96:2310-2313.

20. Downward, J., Graves, J., and Cantrell, D. 1992. The regulation and function of $\mathrm{p} 21$ ras in T cells. Immunol. Today. 13:89-92.

21. Roose, J., and Weiss, A. 2000. T cells: getting a GRP on Ras. Nat. Immunol. 1:275-276.

22. Haneline, L.S., et al. 1999. Loss of Fanc C function results in decreased hematopoietic stem cell repopulating ability. Blood. 94:1-8.

23. Harrison, D. 1980. Competitive repopulation: a new assay for long-term stem cell functional capacity. Blood. 55:77-81.

24. Harrison, D., Astle, C., and Delaittre, J. 1978. Loss of proliferative capacity in immunohemopoietic stem cells caused by serial transplantation rather that aging. J. Exp. Med. 172:1526-1531.

25. Mahgoub, N., et al. 1999. Myeloid malignancies induced by alkylating agents in Nf1 mice. Blood. 93:3617-3623.

26. Shannon, K., et al. 1992. Monosomy 7 myeloproliferative disease in children with neurofibromatosis, type 1: epidemiology and molecular analysis. Blood. 79:1311-1318.

27. Miles, D.K., et al. 1996. Patterns of hematopoietic lineage involvement in children with neurofibromatosis type 1 and malignant myeloid disorders. Blood. 88:4314-4320.

28. Reilly, K.M., Loisel, D.A., Bronson, R.T., McLaughlin, M.E., and Jacks, T. 2000. Nf1;Trp53 mutant mice develop glioblastoma with evidence of strain-specific effects. Nat Genet. 26:109-113.

29. Bader, J.L., and Miller, R.B. 1978. Neurofibromatosis and childhood leukemia. J. Pediatr. 92:925-929.

30. Stiller, C.A., Chessells, J.M., and Fitchett, M. 1994. Neurofibromatosis and childhood leukaemia/lymphoma: a population-based UKCCSG study. Br. J. Cancer. 70:969-972.

31. Largaespada, D.A. 2000. Neurofibromin, the product of the NF1 tumor suppressor gene, regulates the apoptotic decision of hematopoietic progenitors. Blood. 96:460a. (Abstr.)

32. Sattler, M., et al. 1999. BCR/ABL directly inhibits expression of SHIP, an SH2-containing polyinositol-5-phosphatase involved in the regulation of hematopoiesis. Mol. Cell. Biol. 19:7473-7480.

33. Di Cristofano, A., Pesce, B., Cordon-Cardo, C., and Pandolfi, P.P. 1998. Pten is essential for embryonic development and tumour suppression. Nat. Genet. 19:348-355.

34. Emanuel, P., Bates, L., Castleberry, R., Gualtieri, R., and Zuckerman, K. 1991. Selective hypersensitivity to granulocyte-macrophage colony-stimulating factor by juvenile chronic myeloid leukemia hematopoietic progenitors. Blood. 77:925-929.

35. Abkowitz, J.L., Golinelli, D., Harrison, D.E., and Guttorp, P. 2000. In vivo kinetics of murine hemopoietic stem cells. Blood. 96:3399-3405.

36. Nucifora, G., Larson, R.A., and Rowley, J.D. 1993. Persistence of the 8:21 translocation in patients with acute myeloid leukemia type M2 in longterm remission. Blood. 82:712-715.

37. Whyatt, D., et al. 2000. An intrinsic but cell-nonautonomous defect in GATA-1-overexpressing mouse erythroid cells. Nature. 406:519-524.

38. Kaneko, Y., et al. 1989. Chromosome pattern in juvenile chronic myelogenous leukemia, myelodysplastic syndrome, and acute leukemia associated with neurofibromatosis. Lenkemia. 3:36-41.

39. Luna-Fineman, S., et al. 1999. Myelodysplastic and myeloproliferative disorders of childhood: a study of 167 patients. Blood. 93:459-466. 